\title{
Performance analysis of distributed ZF beamforming in the presence of CFO
}

\author{
Yann YL Lebrun ${ }^{1,2^{*}}$, Kanglian KZ Zhao ${ }^{3}$, Sofie SP Pollin ${ }^{2}$, Andre AB Bourdoux ${ }^{2}$, Francois FH Horlin ${ }^{1,4}$ and \\ Rudy RL Lauwereins ${ }^{1,2}$
}

\begin{abstract}
We study the effects of residual carrier frequency offset (CFO) on the performance of the distributed zero-forcing (ZF) beamformer. Coordinated transmissions, where multiple cells cooperate to simultaneously transmit toward one or multiple receivers, have gained much attention as a means to provide the spectral efficiency and data rate targeted by emerging standards. Such schemes exploit multiple transmitters to create a virtual array of antennas to mitigate the co-channel interference and provide the gains of multi-antenna systems. Here, we focus on a distributed scenario where the transmit nodes share the same data but have only the local knowledge of the channels. Considering the distributed nature of such schemes, time/frequency synchronization among the cooperating transmitters is required to guarantee good performance. However, due to the Doppler effect and the non-idealities inherent to the local oscillator embedded in each wireless transceiver, the carrier frequency at each transmitter deviates from the desired one. Even when the transmitters perform frequency synchronization before transmission, a residual CFO is to be expected that degrades the performance of the system due to the in-phase misalignment of the incoming streams. This paper presents the losses of the signal-to-noise ratio gain analytically and the diversity order semi-numerically of the distributed ZF beamformer for the ideal case and in the presence of a residual CFO. We illustrate our results and their accuracy through simulations.
\end{abstract}

Keywords: distributed/coordinated beamforming, carrier frequency offset; residual carrier frequency offset, signalto-noise ratio gain; zero-forcing precoder; diversity order

\section{Introduction}

Coordinated transmissions, where multiple cells cooperate to transmit simultaneously toward one or multiple receivers, have gained much attention recently as a means to provide the spectral efficiency and data rate targeted by emerging standards $[1,2]$. Such schemes create a virtual array of antennas to provide the gains of multi-antenna systems and aid in mitigating the interference in cellular networks [3]. They have the potential to improve the performance or the per-user capacity of the users at the cell edge. This benefits the overall network performance at a low cost, i.e., no need for new infrastructures or expensive devices.

In coordinated transmissions, the beamforming weights are chosen according to the level of knowledge

\footnotetext{
* Correspondence: lebrun.y@gmail.com

'Department of Electrical Engineering, Katholieke Universiteit Leuven,

Kasteel-park Arenberg 10, B-3001 Leuven, Belgium

Full list of author information is available at the end of the article
}

available at each transmitter, i.e., the data and channel state information (CSI), and the degree of cooperation between the transmit cells. The exchange of full CSI and data information between the transmit cells enables the joint computation of the beamforming weights [4]. However, even if this scheme achieves optimal performance, it requires a central coordinator to gather all CSI to jointly compute the beamforming weights and then to redistribute these weights to each transmit cell [5]. The implementation of coordinated transmissions in a distributed network is hence challenging due to the complexity of the joint beamforming and the extensive sharing of information between the transmit cells where backhaul limitations and latency issues arise $[6,7]$. In addition, considering a source broadcasting its symbol information to two relay stations, the symbol information is then readily available at both relays $[8,9]$. However, in such a case, the sharing of the CSI is difficult, 
especially when the receivers are moving and their channels are varying.

Conversely, distributed (yet coordinated) beamforming schemes where each cell exploits the knowledge of the information data but only a limited knowledge about the channels and other transmitter weights are a more practical alternative [6]. Additionally, distributed beamformers are computationally less intensive than their fully coordinated counterpart since they only require the local processing of the beamforming weights. Besides the difficult exchange of the data and CSI between the transmit cells, coordinated systems require perfect synchronization between different cells; this is also challenging to achieve.

Carrier frequency offset (CFO) is caused by the mobility of the wireless devices (Doppler effect) and by the non-ideality of the local oscillator embedded in each wireless transceiver. CFO is a major source of impairment in orthogonal frequency division modulation (OFDM) schemes and must be compensated to obtain acceptable performance [10]. In point-to-point communication, the carrier frequency mismatch causes signalto-noise ratio (SNR) loss, a phase rotation of the symbols and intercarrier interference (ICI). In coordinated communications, each stream originates from a distinct source, each with a different frequency error. As a result, the receiver needs to cope with multiple CFOs and the impacts of CFO in coordinated schemes are hence worse than for point-to-point communications. Because the frequency offset translates into the possibly destructive combination of the incoming streams, it is impossible to correct the multiple CFOs at the receiver. The primary method for mitigating the effects of CFO consists then in compensating the frequency offset before transmission, i.e., it must be corrected by each source $[11,12]$. Methods to estimate the multiple CFOs at the receiver, which requires a different approach compared to point-to-point communications, have also been proposed [13-15].

In practical scenarios, the perfect synchronization of the wireless devices is very challenging and a residual CFO is to be expected even after synchronization [16]. It is therefore of interest to understand the impacts of residual CFO on coordinated communications. Earlier work focused on the results of residual CFO on the bit error rate (BER) performance for cooperative space-frequency/ block code systems $[17,18]$. In addition, simulation results exist on the impacts of CFO in cooperative multiuser MIMO systems [19]. Zarikoff also shows that in multiuser systems the CFOs degrade the accuracy of the beamformer, hence decreasing the capacity [20]. Mudumbai et al., consider a cluster of single-antenna sensor nodes communicating with a distant receiver, where the sensor nodes share a consistent carrier signal
[12]. They identify the time-varying phase drift from the oscillator to dominate the performance degradation and study the resulting SNR loss. Works also include the study of the beamforming gain degradation caused by phase offset estimation errors [21]. These results are complementary to the results presented here. While they study the impacts of phase noise and phase drift in distributed systems, we consider the negative impacts of the time- and CFO-dependent phase mismatch of the incoming streams on the SNR gain and diversity order. Derivations of the SNR and diversity gains without CFO are well known for single-user (SU) scenarios [22] and have also been proposed for amplify-and-forward scenarios [23-25], which are different scenarios to the one we consider in this work. To the best of our knowledge, literature does not evaluate the effects of residual CFO on the SNR gain and diversity order for distributed beamforming schemes where the transmitters share the same time and frequency resources for transmitting a common data toward multiple receivers. For the scenario of interest in this work, no analytical or simulation results exist.

In this paper, we study the effects of a residual CFO on the performance of the distributed zero-forcing (ZF) beamforming scheme, i.e., where both transmitters simultaneously transmit a shared data toward both receivers while suppressing the co-channel interference. We first introduce the system model and derive analytically the SNR gain and the diversity order numerically in the ideal case, i.e., assuming perfect synchronization. Next, we propose the derivations of the SNR gain and diversity order when residual CFO is present. We show that the performance decreases with time as the residual CFO introduces a misalignment of the incoming streams. Finally, simulation results confirm the analytical derivations.

The outline is as follows: In Section 2, the system model of the considered coordinated transmission scheme with perfect synchronization is introduced. The derivations of the average SNR gain and the diversity order are given in Section 3. In Section 4, the system model is defined for multiple CFOs from different transmitters and the derivations for the average SNR and diversity gains with CFO are presented. Simulations in Section 5 show the performance of the cooperative scheme for both the ideal case and when residual CFO is present. These results are discussed together with the proposed analytical derivations. Section 6 concludes our work.

Notations: The following notations are used: The vectors and matrices are in boldface letters, vectors are denoted by lower-case and matrices by capital letters. The superscript $(\cdot)^{H}$ denotes the Hermitian transpose operator, and $(\cdot)^{\dagger}$ denotes the pseudo-inverse. $E[\cdot]$ is the expectation operator, $\mathbf{I}_{N}$ is an identity matrix of size ( $N$ $\times N)$ and $\mathbb{C}^{N \times 1}$ denotes the set of complex vectors of size $(N \times 1)$. The definition $\mathbf{x} \sim \mathbb{C} N\left(\mathbf{0}, \sigma^{2} \mathbf{I}_{N}\right)$ means that 
the vector $\mathbf{x}$ of size $N \times 1$ has zero-mean Gaussian distributed independent complex elements with variance $\sigma^{2}$. We define $\mathbf{a}^{n}$ as the $n^{\text {th }}$ element of the vector $\mathbf{a}$.

\section{System model}

We consider a distributed beamforming system where two independent nodes transmit simultaneously to two receivers. Figure 1 shows the system model. Although the derivations are proposed for a scenario with two transmitters and receivers, they can be generalized to scenarios involving more transmitters and receivers. We assume that the transmitters share information about the data to transmit and that the network protocol guarantees them to be time synchronized. Each transmitter is equipped with $N_{t} \geq 2$ transmit antennas, while the receiver has a single antenna. We assume flat fading channels and present the derivations for the single carrier case. However, assuming only a residual CFO, the impacts of the intercarrier interference (ICI) and SNR loss introduced by the CFO mismatch on a multi-carrier system are negligible compared to the negative impact of phase offset, i.e., the proposed derivations are also valid for a multi-carrier system.

We consider that a prior-to-transmission frequency synchronization is performed so that only a residual CFO is present at the receivers. The initial phase error of the local oscillator at the transmitter side creates a phase error when down (up) converting the receive (transmit) signal. However, this phase error is included in the channel response when estimating the channel. Since the beam-forming weights are computed based on the channel estimates, the beamformer compensates also for this phase error. As a result, this initial phase error can be omitted $[15,26]$.
The channel vector is composed of independent and identically distributed (i.i.d.) Rayleigh fading elements of unit variance: $\mathbf{h}_{i k} \sum \mathbb{C N}\left(\mathbf{0}, \mathbf{I}_{N_{t}}\right)$. It models the $N_{t}$ channels between receiver $i$ and transmitter $k$ with $i, k=1,2$. We denote by $s_{i} \in \mathbb{C}^{1 \times 1}$ the transmitted symbol to the receiver $\operatorname{Rx}_{i}$ where $E_{s_{i}}\left[\left|s_{i}\right|^{2}\right]=1$. Each transmitter exploits only a limited channel knowledge to compute the beamforming weights: each transmitter has only the knowledge of the channels from its own antennas to both receivers, i.e., $\mathrm{Tx}_{1}$ has the channel knowledge of $\mathbf{h}_{11}^{H}$ and $\mathbf{h}_{21}^{H}$, and $\mathrm{Tx}_{2}$ has the channel knowledge of $\mathbf{h}_{22}^{H}$ and $\mathbf{h}_{12}^{H}$. As a result, only the local computation of the beamforming weights is achievable. At the channel input, the transmit signals from $\mathrm{Tx}_{i}, i=1,2$ are denoted by $\mathbf{x}_{i} \in \mathbb{C}^{1 \times 1}$ and can be expressed as

$$
\begin{aligned}
& \mathbf{x}_{1}=\mathbf{w}_{11} s_{1}+\mathbf{w}_{12} s_{2} \\
& \mathbf{x}_{2}=\mathbf{w}_{22} s_{2}+\mathbf{w}_{21} s_{1}
\end{aligned}
$$

where $\mathbf{w}_{i 1} \in \mathbb{C}^{N_{t} \times 1}$ denotes the beamforming vector from the transmitter $i$ toward the $l^{t h}$ receiver. The beamforming vectors satisfy the following power constraint

$$
\mathbf{w}_{\mathrm{i} 1}^{H} \mathbf{w}_{\mathrm{i} 1} \leq P_{i} \quad i=1,2 \quad l=1,2 .
$$

$P_{i}$ denotes the transmit power dedicated to each receiver at $\mathrm{Tx}_{i}$ (a given transmitter allocates the transmit power evenly to both receivers). At the channel output, the received signals at $\mathrm{Rx}_{i}$ are denoted by $y_{i} \in \mathbb{C}^{1 \times 1}$ and can be expressed as

$$
\begin{aligned}
& y_{1}=\left(\mathbf{h}_{11}^{H} \mathbf{w}_{11}+\mathbf{h}_{12}^{H} \mathbf{w}_{21}\right) s_{1}+\left(\mathbf{h}_{11}^{H} \mathbf{w}_{12}+\mathbf{h}_{12}^{H} \mathbf{w}_{22}\right) s_{2}+n \\
& y_{2}=\left(\mathbf{h}_{21}^{H} \mathbf{w}_{12}+\mathbf{h}_{22}^{H} \mathbf{w}_{22}\right) s_{1}+\left(\mathbf{h}_{21}^{H} \mathbf{w}_{11}+\mathbf{h}_{22}^{H} \mathbf{w}_{21}\right) s_{1}+n
\end{aligned}
$$

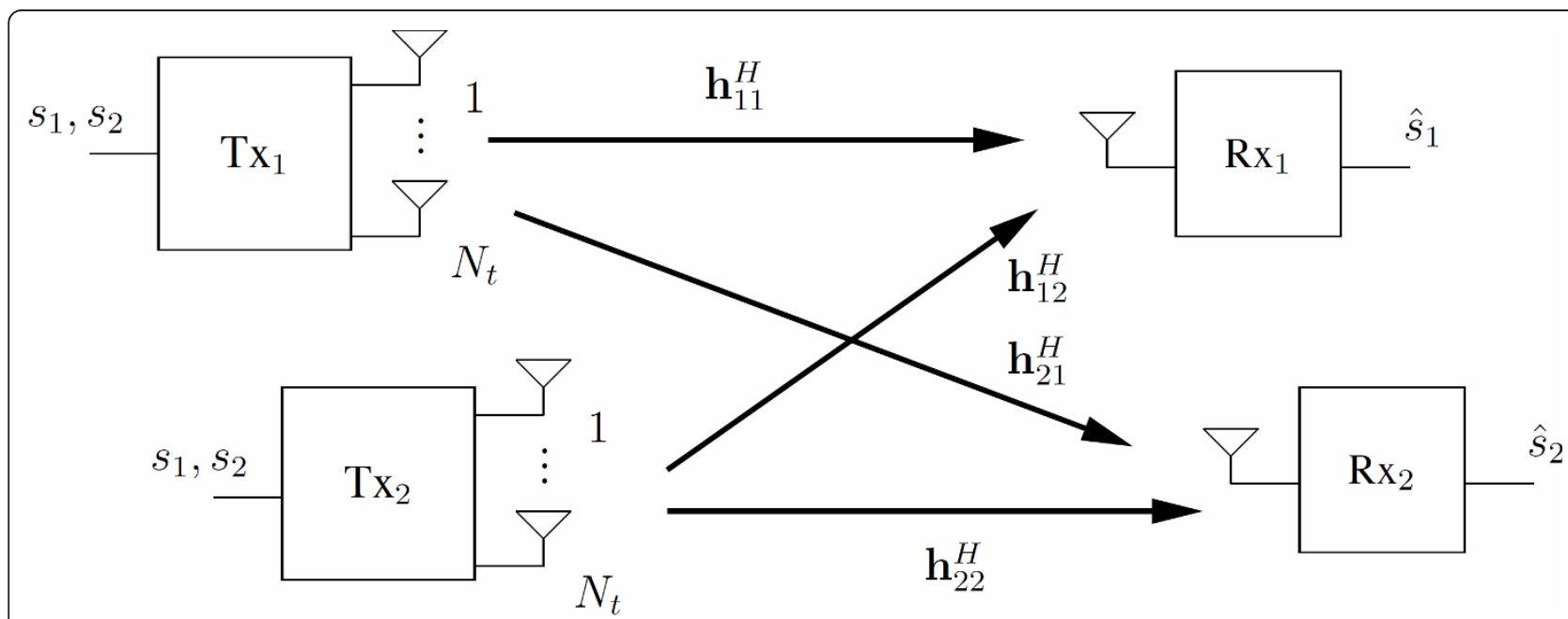

Figure 1 System model of a coordinated scheme in flat fading channels where both transmitters communicate simultaneously toward both receivers. 
where the term $n \in \mathbb{C}^{1 \times 1}$ is the zero-mean circularly symmetric complex additive white Gaussian noise (AWGN) with variance $\sigma_{n}^{2}$.

We consider a ZF beamformer. Such a beamformer exploits the knowledge of the channels from its own antennas to choose the beamforming vector that maximizes the energy while placing the nulls in the direction of the non-targeted user. The computation of the beamforming weights can be decomposed into two steps: null beamforming and maximal energy beamforming. We focus on the computation of the weights for $\mathrm{Tx}_{1}$, and a similar approach can be done for $\mathrm{Tx}_{2}$.

\subsubsection{Null beamforming}

To cancel the interference toward the non-targeted user, the matrix $Z_{i j} \in \mathbb{C}^{N_{t} \times N_{t}}$ is used as the orthogonal projection onto the orthogonal complement of the column space of the channel $\mathbf{h}_{i j}$, e.g., from $\mathrm{Tx}_{1}$ to cancel interference toward $\mathrm{Rx}_{1}$ and $\mathrm{Rx}_{2}$

$$
\begin{aligned}
& \mathbf{Z}_{11}=\mathbf{I}_{N_{t}}-\mathbf{h}_{11}\left(\mathbf{h}_{11}^{H} \mathbf{h}_{11}\right)^{-1} \mathbf{h}_{11}^{H} \\
& \mathbf{Z}_{21}=\mathbf{I}_{N_{t}}-\mathbf{h}_{21}\left(\mathbf{h}_{21}^{H} \mathbf{h}_{21}\right)^{-1} \mathbf{h}_{21}^{H} .
\end{aligned}
$$

\subsubsection{Maximum-ratio combining}

The transmit maximum-ratio combining (MRC) beamformer is applied toward the targeted user [27]. The weights are chosen from the complementary space of the projection matrix to maximize the energy toward the receiver

$$
\mathbf{w}_{11}=\sqrt{P_{1}} \frac{\mathbf{Z}_{21} \mathbf{h}_{11}}{\left\|\mathbf{Z}_{21} \mathbf{h}_{11}\right\|} \quad \text { and } \quad \mathbf{w}_{12}=\sqrt{P_{1}} \frac{\mathbf{Z}_{11} \mathbf{h}_{21}}{\left\|\mathbf{Z}_{11} \mathbf{h}_{21}\right\|}
$$

which fulfills the power constraint in (2). Since the ZF beamforming weights lay in the null space of the nontargeted user, the received signal is interference free.

Equations in (3) can be written as

$$
\begin{aligned}
& y_{1}=\left(\mathbf{h}_{11}^{H} \mathbf{w}_{11}+\mathbf{h}_{12}^{H} \mathbf{w}_{21}\right) s_{1}+n \\
& y_{2}=\left(\mathbf{h}_{21}^{H} \mathbf{w}_{12}+\mathbf{h}_{22}^{H} \mathbf{w}_{22}\right) s_{2}+n .
\end{aligned}
$$

We have expressed the transmit and received signals and defined the beamforming weights for the considered scheme. In the next section, we derive the resulting SNR and diversity gains assuming perfect synchronization, $i$. e., no CFO.

\section{SNR and diversity gains}

The SNR gain comes from the (coherent) addition of the incoming streams at the receiver antennas. It is obtained by averaging the instantaneous SNR over the channel realizations and indicates the SNR gain over the single-user (SU) single-input-single-output (SISO) case. We derive the resulting average SNR (Section 3.1) and to compare it to the SNR gain in SU scenarios. The diversity gain is obtained by combining the multiple replicas of the signal collected at the receiver. The diversity order is calculated by evaluating the resulting slope of the average bit error rate curve, and the derivation of the diversity order is proposed in Section 3.2.

\subsection{Average SNR gain}

The instantaneous SNR denotes the power of the received signal, after equalization, averaged over the noise and symbols. In the following derivations, we assume a zero-forcing (ZF) complex scalar equalizer at the receiver, i.e., the inversion of the equivalent channel. For the sake of clarity, the derivations are performed for $\mathrm{Rx}_{1}$ only. From Equation (6), after processing at the receiver, the estimated symbol can be expressed as

$$
\begin{aligned}
y_{1} & =\left(\mathbf{h}_{11}^{H} \mathbf{w}_{11}+\mathbf{h}_{12}^{H} \mathbf{w}_{21}\right)^{-1}\left(\left(\mathbf{h}_{11}^{H} \mathbf{w}_{11}+\mathbf{h}_{12}^{H} \mathbf{w}_{21}\right) s_{1}+n\right) \\
& =s_{1}+\left(\mathbf{h}_{11}^{H} \mathbf{w}_{11}+\mathbf{h}_{12}^{H} \mathbf{w}_{21}\right)^{-1} n=s_{1}+e_{1} .
\end{aligned}
$$

We denote the symbol error by $e_{1}=\left(\mathbf{h}_{11}^{H} \mathbf{w}_{11}+\mathbf{h}_{12}^{H} \mathbf{w}_{21}\right)^{-1} n$. We then obtain the following instantaneous output SNR from $\mathrm{Rx}_{1}$ for one channel realization $\left(\gamma_{1}\right)$ by taking the expectations over the noise and the symbols

$$
\gamma_{1}=\frac{1}{E\left[\left(e_{1}\right)^{2}\right]}=\frac{1}{\sigma_{n}^{2}}\left(\mathbf{h}_{11}^{H} \mathbf{w}_{11}+\mathbf{h}_{12}^{H} \mathbf{w}_{21}\right)^{2} .
$$

Next, we average $\gamma_{1}$ over the channel realizations to obtain the average SNR

$$
\begin{aligned}
& E\left[\gamma_{1}\right]=\frac{1}{\sigma_{n}^{2}} E\left[\left(\mathbf{h}_{11}^{H} \mathbf{w}_{11}+\mathbf{h}_{12}^{H} \mathbf{w}_{21}\right)^{2}\right] \\
& =\frac{1}{\sigma_{n}^{2}}\left(E\left[\left(\mathbf{h}_{11}^{H} \mathbf{w}_{11}\right)^{2}\right]+E\left[\left(\mathbf{h}_{12}^{H} \mathbf{w}_{21}\right)^{2}\right]+2 E\left[\mathbf{h}_{11}^{H} \mathbf{w}_{11}\right] E\left[\mathbf{h}_{12}^{H} \mathbf{w}_{21}\right]\right)
\end{aligned}
$$

From the results in (5), the combination of the precoder with the channel, e.g., $\mathbf{h}_{11}^{H} \mathbf{w}_{11}$, gives

$$
\mathbf{h}_{11}^{H} \mathbf{w}_{11}=\sqrt{P_{1}} \frac{\mathbf{h}_{11}^{H} \mathbf{Z}_{21} \mathbf{h}_{11}}{\left\|\mathbf{Z}_{21} \mathbf{h}_{11}\right\|}=\sqrt{P_{1}} \frac{\mathbf{h}_{11}^{H} \mathbf{Z}_{21} \mathbf{h}_{11}}{\sqrt{\left(\mathbf{h}_{11}^{H} \mathbf{Z}_{21}^{H} \mathbf{Z}_{21} \mathbf{h}_{1,1}\right)}}
$$

If the matrix $\mathbf{Z}$ is a projection matrix (Equation (4)), it is idempotent: $\mathbf{Z}=\mathbf{Z}^{2}$ [28]. 
We can then write $\mathbf{h}_{11}^{H} \mathbf{Z}_{21}^{H} \mathbf{Z}_{21} \mathbf{h}_{11}=\mathbf{h}_{11}^{H} \mathbf{Z}_{21} \mathbf{h}_{11}$, i.e.,

$$
\mathbf{h}_{11}^{H} \mathbf{w}_{11}=\sqrt{P_{1}\left(\mathbf{h}_{11}^{H} \mathbf{Z}_{21} \mathbf{h}_{11}\right)} \text {. }
$$

Next, applying the eigenvalue decomposition to the matrix $\mathbf{Z}_{21}$, we obtain

$$
\mathbf{h}_{11}^{H} \mathbf{Z}_{21} \mathbf{h}_{11}=\mathbf{h}_{11}^{H} \mathbf{U}_{21} \mathbf{\Lambda}_{21} \mathbf{U}_{21}^{H} \mathbf{h}_{11} .
$$

The matrix $\mathbf{U}_{21}$ is a unitary matrix of eigenvectors, and $\boldsymbol{\Lambda}_{21}$ is a diagonal matrix containing the eigenvalues. Because the properties of a zero-mean complex Gaussian vector do not change when multiplied with a unitary matrix, we have $\mathbf{h}_{11}^{H} \mathbf{U} \sim \mathbf{h}_{11}^{H}$. From the results above, we obtain

$$
E\left[\mathbf{h}_{11}^{H} \mathbf{w}_{11}\right]=E\left[\sqrt{P_{1}\left(\mathbf{h}_{11}^{H} \mathbf{\Lambda}_{21} \mathbf{h}_{11}\right)}\right] .
$$

Again, the matrix $\mathbf{Z}_{21}$ being idempotent, its eigenvalues are either 1 or 0 [28]. As a result, the rank of $\mathbf{Z}_{21}$ equals its trace

$$
\begin{aligned}
\operatorname{rank}\left(\mathbf{Z}_{i j}\right) & =\operatorname{tr}\left(\mathbf{I}_{N_{t}}-\mathbf{h}_{i j}\left(\mathbf{h}_{i j}^{H} \mathbf{h}_{i j}\right)^{-1} \mathbf{h}_{i j}^{H}\right) \\
& =\operatorname{tr}\left(\mathbf{I}_{N_{t}}\right)-\operatorname{tr}\left(\mathbf{h}_{i j}\left(\mathbf{h}_{i j}^{H} \mathbf{h}_{i j}\right)^{-1} \mathbf{h}_{i j}^{H}\right)=N_{1}-1 .
\end{aligned}
$$

The term $E\left[\mathbf{h}_{11}^{H} \mathbf{w}_{11}\right]$ can then equivalently be expressed as

$$
E\left[\mathbf{h}_{11}^{H} \mathbf{w}_{11}\right]=E\left[\sqrt{P_{1} \sum_{n=1}^{N_{t}-1}\left|\mathbf{h}_{11}^{n}\right|^{2}}\right] .
$$

From Equation (16), we then have

$$
E\left[\left|\mathbf{h}_{11}^{H} \mathbf{w}_{11}\right|^{2}\right]=E\left[P_{1} \sum_{n=1}^{N_{t}-1}\left|\mathbf{h}_{11}^{n}\right|^{2}\right] .
$$

As a result, we can write Equation (10) as

$$
\begin{aligned}
& E\left[\gamma_{1}\right]=\frac{1}{\sigma_{m}^{2}}\left(P_{1} E\left[\sum_{n=1}^{N_{t}-1}\left|\mathbf{h}_{11}^{n}\right|^{2}\right]+P_{2} E\left[\sum_{n=1}^{N_{t}-1}\left|\mathbf{h}_{12}^{n}\right|^{2}\right]\right. \\
& \left.+2 E\left[\sqrt{P_{1} \sum_{n=1}^{N_{t}-1}\left|\mathbf{h}_{11}^{n}\right|^{2}}\right] \times E\left[\sqrt{P_{2} \sum_{n=1}^{N_{t}-1}\left|\mathbf{h}_{12}^{n}\right|^{2}}\right]\right) .
\end{aligned}
$$

From this equation, $\sqrt{\sum_{n=1}^{N_{t}-1}\left|\mathbf{h}_{11}^{n}\right|^{2}}$ is a Rayleigh distributed random variable [29]

$$
E\left[\sqrt{\sum_{n=1}^{N_{t}-1}\left|\mathbf{h}_{11}^{n}\right|^{2}}\right]=\frac{\Gamma\left(N_{1}-0.5\right)}{\left(N_{t}-2\right) !}
$$

where $\Gamma$ denotes the Gamma function and $(N)$ ! the factorial of $N$. We can recognized that the expression $\left|\mathbf{h}_{11}^{n}\right|^{2}$ follows a chi-square distribution [29], and we hence obtain

$$
E\left[\sum_{n=1}^{N_{t}-1}\left|\mathbf{h}_{11}^{n}\right|^{2}\right]=\frac{\left(\Gamma N_{t}\right)}{\left(N_{1}-2\right) !}=N_{t}-1 .
$$

Finally, the average SNR (in $\mathrm{dB}$ ) for the distributed ZF scheme assuming perfect synchronization can be expressed as

$$
G=10 \log _{10}\left(\left(P_{1}+P_{2}\right)\left(N_{1}-1\right)+2 \sqrt{P_{1} P_{2}}\left(\frac{\Gamma\left(N_{t}-0.5\right)}{\left(N_{t}-2\right)}\right)^{2}\right) .
$$

For comparison, the SNR gain for the single-user case, with a transmit MRC beamformer, is

$$
G_{M R C}=10 \log _{10}\left(P N_{t}\right)
$$

while for the equal gain combining (EGC) beamformer [22], it is given as

$$
G_{E G C}=10 \log _{10}\left(P\left(1+\left(N_{t}-1\right) \frac{\pi}{4}\right)\right) .
$$

From these results, with $P=P_{1}+P_{2}\left(P_{1}=P_{2}\right)$ and $N_{t}$ $=2$, the SNR of the ZF coordinated and EGC schemes is equal. This is expected since the two cells transmit with equal power and because one degree of freedom is used by the ZF scheme to cancel interference. However, with $N_{t} \geq 3$, the SNR gain of the ZF coordinated scheme outperforms the EGC and MRC beamformers, i.e., $G=$ $8.77 \mathrm{~dB}$ while $G_{\mathrm{MRC}}=7.78 \mathrm{~dB}$ and $G_{\mathrm{EGC}}=7.1 \mathrm{~dB}$.

\subsection{Diversity order}

The diversity gain is obtained by combining the multiple replicas of the signal collected at the receiver. The diversity order is calculated by evaluating the resulting slope of the average bit error rate curve.

The diversity order for the first receiver is given as

$$
-d_{1}=\lim _{\sigma_{n}^{2} \rightarrow \infty} \frac{\log _{10} P_{e}}{\log _{10} \sigma_{n}^{2}}
$$

where $P_{e}$ denotes the average bit error rate probability for the first receiver

$$
P_{e}=\int_{0}^{\infty} P_{c}\left(e \mid \gamma_{1}\right) p_{\gamma_{1}}\left(\gamma_{1}\right) d \gamma_{1} .
$$

We denote by $p_{\gamma_{1}}\left(\gamma_{1}\right)$ the probability density function (PDF) of the instantaneous SNR $\left(\gamma_{1}\right)$ at the receiver 1 given in Equation (8). The expression $P_{c}\left(e \mid \gamma_{1}\right)$ denotes the conditional bit error rate and can be expressed, for a binary phase-shift keying (BPSK) modulation, as 


$$
P_{c}\left(e \mid \gamma_{1}\right)=Q\left(\sqrt{2 \gamma_{1}}\right) \text {. }
$$

where $Q(x)$ denotes the alternative Gaussian $Q$ function representation [22] given as

$$
Q(x)=\frac{1}{\pi} \int_{0}^{\frac{\pi}{2}} \exp \left(-\frac{x^{2}}{2 \sin ^{2} \phi}\right) d \phi
$$

hence $P_{c}\left(e \mid \gamma_{1}\right)=\frac{1}{\pi} \int_{0}^{\frac{\pi}{2}} \exp \left(-\frac{2 \gamma_{1}}{2 \sin ^{2} \phi}\right) d \phi$. We can write the average bit error rate probability as

$$
P_{e}=\int_{0}^{\infty} \frac{1}{\pi} \int_{0}^{\frac{\pi}{2}} \exp \left(-\frac{2 \gamma_{1}}{2 \sin ^{2} \phi}\right) d \phi p_{\gamma_{1}}\left(\gamma_{1}\right) d \gamma_{1} .
$$

Developing the equation of the instantaneous SNR in Equation (8) gives

$$
\gamma_{1}=\frac{1}{\sigma_{m}^{2}}\left(\left(\mathbf{h}_{11}^{H} \mathbf{w}_{11}\right)^{2}+\left(\mathbf{h}_{12}^{H} \mathbf{w}_{21}\right)^{2}+2 \mathbf{h}_{11}^{H} \mathbf{w}_{11} \mathbf{h}_{12}^{H} \mathbf{w}_{21}\right)
$$

Because the terms in (29) are not independent, obtaining the equivalent PDF is hence difficult. In this case, we take the square root of the instantaneous SNR, i.e.,

$$
\eta=\sqrt{\gamma_{1}} .
$$

This is a sum of chi-random variables (RVs) where each chi-RV has $2\left(N_{t}-1\right)$ degrees of freedom. From the results in [30], we can express the equivalent $\operatorname{PDF} p_{\eta}(\eta)$ as follows

$$
p_{n}(\eta)=\frac{4 e^{-\eta^{2} / 4}}{2^{N_{t}-1}\left[\Gamma\left(\frac{N_{t}-1}{2}\right)\right]^{2}} \sum_{r=0}^{N_{t}-2}(-1)^{r} \eta^{N_{t}-2-r} a_{0}(r)
$$

where

$$
a_{0}(r)=\int_{0}^{\infty} x^{N_{t}-2+r} e^{-(x-\eta / 2)^{2}} .
$$

However, no general closed form of the equivalent PDF can be obtained. Therefore, we compute the integral in Equation (28) numerically and for a varying number of antennas. These numerical approximations of the error probability Pe are then used to compute the diversity order of the considered cooperative scheme. With two antennas at each transmitter, each chi-random variable has $2\left(N_{t}-1\right)=2$ degrees of freedom. Because a chi-RV with two degrees of freedom has a Rayleigh distribution, the diversity order is then equivalent to a single-user EGC scenario with two antennas and provides then a diversity order of 2 [31]. From the numerical analysis and simulation results in Section 5.1, we recognize a diversity order of $2\left(N_{t}-1\right)$. This result should be expected as one degree of freedom cancels the interference toward the non-intended receiver. A similar approach can be employed for the second receiver.

\section{Distributed ZF beamforming: impact of CFO}

From the results given in the Section 2, good performance is expected from the distributed ZF beamformer thanks to the added SNR and diversity gains. In this section, we discuss the effects of the residual CFO on those gains. In 4.1, we extend the system model given in Section 2 to the case where CFO is present. Then, the average SNR gain and diversity order are derived for the general case ( $N_{t}$ transmit antennas) in Sections 4.2 and 4.3 .

\subsection{System model with CFOs}

The combination of the channel with the carrier frequency offset can be equivalently represented by the channel vector multiplied by the complex component $c_{i}\left(t, f_{\Delta_{i}}\right)=e^{\phi_{i}\left(t, f_{\Delta_{i}}\right)}=e^{i 2 \pi f_{\Delta_{i}} t}$, where $t$ is the time index and $f_{\Delta_{i}}$ denotes the CFO at the transmitter $i$ with respect to the receiver's carrier frequency. Because of the CFO, the time coherency of the channel reduces, and the originally quasi-static channel now becomes time varying hence decreasing the performance of the beamforming scheme with static weights. As introduced earlier, we assume that the frequency offset is precompensated at the transmitters prior to transmission, i.e., only the residual CFOs $f_{\Delta_{1}}$ and $f_{\Delta_{2}}$ are left. We assume no initial phase offset between the transmitters. Equation (8), giving the instantaneous output SNR $\xi_{1}\left(t, f_{\Delta_{1}}, f_{\Delta_{2}}\right)$, can be written as follows

$$
\begin{aligned}
& \frac{1}{\sigma_{n}^{2}}\left(c_{1}\left(t, f_{\Delta_{1}}\right) \mathbf{h}_{11}^{H} \mathbf{w}_{11}+c_{2}\left(t, f_{\Delta_{2}}\right) \mathbf{h}_{12}^{H} \mathbf{w}_{21}\right)^{2} \\
=\frac{1}{\sigma_{n}^{2}}\left(\left(\mathbf{h}_{11}^{H} \mathbf{w}_{11}\right)^{2}+\left(\mathbf{h}_{12}^{H} \mathbf{w}_{21}\right)^{2}+c_{1}\left(t, f_{\Delta_{1}}\right) c_{2}\left(t, f_{\Delta_{2}}\right)^{H} \mathbf{h}_{11}^{H} \mathbf{w}_{11}\left(\mathbf{h}_{12}^{H} \mathbf{w}_{21}\right)^{H}\right. & \left.\quad+c_{1}\left(t, f_{\Delta_{1}}\right)^{H} c_{2}\left(t, f_{\Delta_{2}}\right)\left(\mathbf{h}_{11}^{H} \mathbf{w}_{11}\right)^{H} \mathbf{h}_{12}^{H} \mathbf{w}_{21}\right)
\end{aligned}
$$

We now average $\xi_{1}$ over the channel realizations $E\left[\xi_{1}\left(t, f_{\Delta_{1}}, f_{\Delta_{2}}\right)\right]$ equals

$$
\begin{aligned}
\frac{1}{\sigma_{n}^{2}} & \left(E\left[\left(\mathbf{h}_{11}^{H} \mathbf{w}_{11}\right)^{2}\right]+E\left[\mathbf{h}_{12}^{H} \mathbf{w}_{21}\right]^{2}+\left(c_{1}\left(t, f_{\Delta_{1}}\right) c_{2}\left(t, f_{\Delta_{2}}\right)^{H}\right.\right. \\
& \left.\left.+c_{1}\left(t, f_{\Delta_{1}}\right)^{H} c_{2}\left(t, f_{\Delta_{2}}\right)\right) E\left[\mathbf{h}_{11}^{H} \mathbf{w}_{11}\right] E\left[\mathbf{h}_{12}^{H} \mathbf{w}_{21}\right]\right) .
\end{aligned}
$$

\subsection{Average SNR gain with CFO 4.2.1 Fixed CFO}

Following the procedure for Equation (18) and from Equation (34), the average SNR is $E E\left[\xi_{1}\left(t, f_{\Delta_{1}}, f_{\Delta_{2}}\right)\right]$ 


$$
\begin{aligned}
=\frac{1}{\sigma_{n}^{2}}( & P_{1} E\left[\sum_{n=1}^{N_{t}-1}\left|\mathbf{h}_{11}^{n}\right|^{2}\right]+P_{2} E\left[\sum_{n=1}^{N_{t}-1}\left|h_{12}^{n}\right|^{2}\right]+\left(c_{1}\left(t, f_{\Delta_{1}}\right) c_{2}\left(t, f_{\Delta_{2}}\right)^{H}\right. \\
& \left.\left.+c_{1}\left(t, f_{\Delta_{1}}\right)^{H} c_{2}\left(t, f_{\Delta_{2}}\right)\right) \sqrt{P_{1} P_{2}} E\left[\sqrt{\sum_{n=1}^{N_{t}-1}\left|\mathbf{h}_{11}^{n}\right|^{2}}\right] E\left[\sqrt{\sum_{n=1}^{N_{t}-1}\left|\mathbf{h}_{12}^{n}\right|^{2}}\right]\right)
\end{aligned}
$$

where $\quad c_{1}\left(t, f_{\Delta_{1}}\right) c_{2}\left(t, f_{\Delta_{2}}\right)^{H}+c_{1}\left(t, f_{\Delta_{1}}\right)^{H} c_{2}\left(t, f_{\Delta_{2}}\right) \quad$ is equivalent to

$$
e^{i 2 \pi f \Delta_{1} t} e^{-i 2 \pi f_{\Delta_{2}} t}+e^{-i 2 \pi f_{\Delta_{1}} t} e^{i 2 \pi f_{\Delta_{2}} t}=2 \cos \left(2 \pi f_{\Delta} t\right), \quad f_{\Delta}=f_{\Delta_{1}}-f_{\Delta_{2}} .
$$

The notation $f_{\Delta}$ refers to the relative CFO between two transmitters. This result shows that the average SNR is time-dependent and it varies over the transmission period $T_{p}$. We then compute the time-averaged result to obtain the exact average SNR

$$
E_{T}\left[\cos \left(2 \pi f_{\Delta} t\right)\right]=\frac{1}{T_{p}} \int_{0}^{T_{p}} \cos \left(2 \pi f_{\Delta} t\right) \mathrm{d} t=\operatorname{sinc}\left(2 \pi f_{\Delta} T_{p}\right)
$$

From Equation (35), we obtain $E\left[\xi_{1}\left(T_{p}, f_{\Delta}\right)\right]$

$$
\begin{aligned}
=\frac{1}{\sigma_{n}^{2}} & \left(P_{1} E\left[\sum_{n=1}^{N_{t}-1}\left|\mathbf{h}_{11}^{n}\right|^{2}\right]+P_{2} E\left[\sum_{n=1}^{N_{t}-1}\left|\mathbf{h}_{12}^{n}\right|^{2}\right]\right. \\
& \left.+2 \operatorname{sinc}\left(2 \pi f_{\Delta} T_{p}\right) \sqrt{P_{1} P_{2}} E\left[\sqrt{\sum_{n=1}^{N_{t}-1}\left|\mathbf{h}_{11}^{n}\right|^{2}}\right] E\left[\sqrt{\sum_{n=1}^{N_{t}-1}\left|\mathbf{h}_{12}^{n}\right|^{2}}\right]\right) .
\end{aligned}
$$

As a result, based on the (19) and Equation (20) and following the procedure in Section 3.1, we can express the average SNR gain of the proposed scheme with residual $\mathrm{CFO}$ as $G_{\text {CFOfixed }}$ equals to

$$
10 \log _{10}\left(\left(P_{1}+P_{2}\right)\left(N_{t}-1\right)+2 \sin c\left(2 \pi f_{\Delta} T_{p}\right) \sqrt{P_{1} P_{2}}\left(\frac{\Gamma\left(N_{t}-0.5\right)}{\left(N_{t}-2\right) !}\right)^{2}\right) .
$$

We can observe that the average SNR gain degrades, following a sinc function with the parameters $f_{\Delta}$ and $T_{p}$. As a result, for a long enough $T_{p}$, the sinc function produces a zero, i.e., no SNR gain is obtained.

\subsubsection{Uniform CFO}

Assuming an uniformly distributed residual frequency offset, we obtain the average SNR by taking the expectation of the sinc function over the random $f_{\Delta}$, i.e.,

$$
E\left[\operatorname{sinc}\left(2 \pi f_{\Delta} T_{p}\right)\right]=\int_{-f_{c}}^{f_{c}} \operatorname{sinc}\left(2 \pi x T_{p}\right) p(x) \mathrm{d} x
$$

where $f_{c}$ denotes the maximal frequency offset and $x$ is a random variable uniformly distributed over the interval $\left[-f_{o} f_{c}\right]$. Equation (40) is hence equivalent to

$$
E\left[\operatorname{sinc}\left(2 \pi f_{\Delta} T_{p}\right)\right]=\int_{-2 \pi T_{p} f_{c}}^{2 \pi T_{p} f_{c}} \operatorname{sinc}(x) p(x) \mathrm{d} x=\frac{1}{2 \pi T_{p} f_{c}} \operatorname{si}\left(2 \pi T_{p} f_{c}\right)
$$

where $S i(x)$ denotes the sine integral. As a result, the SNR gain with an uniformly distributed CFO in the interval $\left[-f_{c}, f_{c}\right]\left(G_{\text {CFOunif }}\right)$ can be expressed as

$$
10 \log _{10}\left(\left(P_{1}+P_{2}\right)\left(N_{t}-1\right)+\frac{s i\left(2 \pi T_{p} f_{c}\right)}{\pi T_{p} f_{c}} \sqrt{P_{1} P_{2}}\left(\frac{T\left(N_{t}-0.5\right)}{\left(N_{t}-2\right) !}\right)^{2}\right)
$$

\subsection{Diversity order}

\subsubsection{Fixed CFO}

As expressed in Section 3.2, the average error probability $P_{e}$ is required to compute the diversity order $d$. However, when a residual CFO is present, the error probability $P_{e}$ becomes time- and CFO-dependent. For $P_{1}=P_{2}=1$, for a given transmit duration and a residual CFO $\left(f_{\Delta}\right)$, the error probability $P_{e}\left(t, f_{\Delta}\right)$ and a BPSK modulation, we have

$$
P_{e}\left(t, f_{\Delta}\right)=\int_{0}^{\infty} Q\left(\sqrt{2 \xi_{1}\left(t, f_{\Delta}\right)}\right) p_{\xi_{1}}\left(\xi_{1}\left(t, f_{\Delta}\right)\right) d \xi_{1}\left(t, f_{\Delta}\right), \quad 0 \leq t \leq T_{p}
$$

where $\xi_{1}\left(t, f_{\Delta}\right)$ denotes the equivalent signal at the time index $T_{p}=t$ and, from Equation (33), can be expressed as

$$
\xi_{1}\left(t, f_{\Delta}\right)=\frac{1}{\sigma_{n}^{2}}\left(\left(\mathbf{h}_{11}^{H} \mathbf{w}_{11}\right)^{2}+\left(\mathbf{h}_{12}^{H} \mathbf{w}_{21}\right)^{2}+2 \cos \left(2 \pi f_{\Delta} t\right) \mathbf{h}_{11}^{H} \mathbf{w}_{11} \mathbf{h}_{12}^{H} \mathbf{w}_{21}\right)
$$

Similar to Section 3.2, we express the average BER as

$$
P_{e}\left(t, f_{\Delta}\right)=\frac{1}{\pi} \int_{0}^{\frac{\pi}{2}} \int_{0}^{\infty} \exp \left(-\frac{2 \xi_{1}\left(t, f_{\Delta}\right)}{2 \sin ^{2} \phi}\right) p_{\xi_{1}}\left(\xi_{1}\left(t, f_{\Delta}\right)\right) d \xi_{1}\left(t, f_{\Delta}\right) d \phi .
$$

Using the characteristic function ( $\mathrm{CHF}$ ) method to evaluate the PDF $p\left(\gamma_{1}\right)$ requires to obtain $\Psi_{\gamma_{1}}(j v)$. Where $\Psi_{x}(j v)$ is the CHF of the random variable $x$

$$
\Psi_{x}(j v)=E\left[e^{j v x}\right]=\int_{-\infty}^{\infty} e^{j v x} p(x) \mathrm{d} x .
$$

Assuming that the transmitters have a same power of 1, i.e., $P_{1}=P_{2}=1$, from Equations (8), (17) and (46), the equivalent $\mathrm{CHF}$ using the $\mathrm{CHF}$ method to evaluate the PDF $p\left(\xi_{1}\left(t, f_{\Delta}\right)\right)$ requires to compute the $\Psi_{\xi_{1}}\left(j v, t, f_{\Delta}\right)$. From Equations (44) and (35), the equivalent CHF $\Psi_{\xi_{1}}\left(j v, t, f_{\Delta}\right)$ can be expressed as

$$
\begin{aligned}
E\left[e^{j \nu_{1}}\right] & =E\left[e^{j \nu}\left(\left(\mathbf{h}_{11}^{H} \mathbf{W}_{11}\right)^{2}+\left(\mathbf{h}_{12}^{H} \mathbf{W}_{21}\right)^{2}+2 \cos \left(2 \pi f_{\Delta} t\right) \mathbf{h}_{11}^{H} \mathbf{W}_{11} \mathbf{h}_{12}^{H} \mathbf{W}_{21}\right)\right] \\
& =E\left[e^{j \nu} \sum_{n=1}^{N_{t}-1}\left|h_{n}\right|^{2} e^{j \nu} \sum_{n=1}^{N_{t}-1}\left|h_{n}\right|^{2 j e^{j \nu} \cos \left(2 \pi f_{\Delta} t\right)} \sqrt{\sum_{n=1}^{N_{t}-1}\left|\mathbf{h}_{11}^{n}\right|^{2}} \sqrt{\sum_{n=1}^{N_{t}-1}\left|\mathbf{h}_{12}^{n}\right|^{2}}\right]
\end{aligned}
$$

However, similar to the results in Section 3.2, the third term in Equation (47) is not independent from the two others and the expectation operator cannot be separated, obtaining the equivalent PDF (and hence $P_{e}$ ) in a general closed form is difficult.

In addition, the average BER must be integrated over the time index $t$ for a given transmission duration 
$\left(T_{p}\right)$. Therefore, to compute the diversity order of the considered cooperative scheme, we approximate numerically the error probability Pe for a varying number of antennas. We then obtain the average probability of error by integrating the different $P_{e}$, i.e., for a given residual CFO $\left(f_{\Delta}\right)$, over the transmission duration

$$
P_{e \mid T_{p}}=\frac{1}{T_{p}} \int_{0}^{T_{p}} P_{e}(t) \mathrm{d} t
$$

We then use these numerical approximations of the error probability Pe to compute the diversity order of the considered cooperative scheme when CFO is present. The Section 5.2 presents the resulting diversity order.

\subsubsection{Uniform CFO}

We study the effects of a random and uniformly distributed CFO on the diversity order. In such a case, the diversity order is obtained by numerically approximating the PDF of the equivalent channel for a random variable $\Delta_{f}$ uniformly distributed over the interval $\left[-f_{c}, f_{c}\right]$. Section 5 presents the results from the diversity order with an uniformly distributed CFO.

\section{Simulation results}

This section aims at comparing the SNR and diversity gains of the distributed ZF beamforming scheme without synchronization errors (Section 2) with respect to the case with residual CFO (Section 4) and verifying the proposed derivations.

As already mentioned in the text, we consider a distributed transmission scenario where two independent cells transmit simultaneously a same data toward two receivers. The simulations are performed for the IEEE802.11 n system [32] with a $5 \mathrm{GHz}$ carrier frequency and a $20 \mathrm{MHz}$ bandwidth. We consider an uncoded OFDM scheme with 64 subcarriers. A power of 1 is allocated from a receiver to each transmitter, $\mathrm{i}$. e., $P_{1}=P_{2}=1$. The multiple CFOs are assumed known at the receiver where a zero-forcing frequency domain equalizer is applied for synchronization. Presynchronization of the frequency offset is performed at the transmitters so that only residual $\mathrm{CFO} f_{\Delta}$ is left. The $f_{\Delta}$ is expressed in part per million (ppm) with respect to the system carrier frequency. We assume the network protocol to guarantee the transmitters to be time synchronized and assume no initial phase offset between the transmitters. Each scenario can be described as $N_{\mathrm{TX}}\left(N_{t}\right) \times N_{\mathrm{RX}}\left(N_{r}\right)$, where $N_{\mathrm{TX}}$ denotes the number of transmitters and $N_{R X}$ the number of receivers, $N_{t}$ is the number of transmit antennas at each transmitter, and $N_{r}$ is the number of antennas at each receiver.
5.1 Performance of cooperative beamforming: ideal case The Figure 2 displays the BER curves versus the SNR, i. e., $1 / \sigma_{n}^{2}$, of the considered ZF beamforming scheme, i.e., the multi-user (MU) scenario, with $N_{t}=2, N_{t}=3$ and $N_{t}=4$ assuming perfect frequency synchronization and for a 16 QAM modulation scheme. The BER curves of a single-user (SU) system with transmit-MRC beamforming and $N_{t}=2$ and $N_{t}=4$ are also displayed. From this figure, we can observe that the diversity order $(d)$ of the considered scheme results in a diversity order for the ZF beamformer with $N_{t}=3$ of $2 \times(3-1)=4$, and this is equivalent to that of the SU transmit-MRC scheme with four transmit antennas. Similarly, the ZF beamforming scheme with $N_{t}=2$ provides the same diversity order $(d=2)$ as the SU scheme with two transmit antennas. The figure also shows that the SNR gain of the ZF beamforming scheme with $N_{t}=2$ is of approximately $0.5 \mathrm{~dB}$ less than that of the $\mathrm{SU}$ scheme with two transmit antennas as expected from Section 2. Similarly, at $10 \mathrm{~dB}$ SNR, the SNR gain of the ZF beamformer with $N_{t}=3$ is of approximately $0.2 \mathrm{~dB}$ less than that of the $\mathrm{SU}$ transmit-MRC scheme with four transmit antennas, as expected from Equation (21).

\subsection{Performance of coordinated beamforming with frequency offset}

Here, we study the effects of residual CFO on the SNR and diversity gains. In the simulations, we assume two transmitters each equipped with two or more antennas, i.e., $N_{t} \geq 2$.

\subsubsection{SNR gain with residual CFO}

From the derivations in Section 3.1, a residual CFO introduces a SNR loss that follows a sinc function. In Figure 3, we display both the analytical (dashed line) and simulated SNR gain for a $f_{\Delta}=2 \mathrm{ppm}, N_{t}=2$, and for various transmit symbols $\left(T_{p}\right)$ for both a fixed and an uniform residual CFO. We can observe that the CFO degrades the SNR gain, for example, approximately 0.5 $\mathrm{dB}$ gain is lost after five OFDM symbols while $2 \mathrm{~dB}$ gain is lost after 10 symbols and a fixed residual CFO.

\subsubsection{Diversity order with residual CFO}

The Figure 4 shows the BER curves for various transmission durations based on the analytical derivations given in Section 4.3. The simulations are for $f_{\Delta}=2 \mathrm{ppm}$ and a BPSK modulation scheme and $N_{t}=3$. From this figure, we can observe that the diversity order decreases quickly with the number of transmit symbols to finally approach the SU-SISO curve for a long transmit period, e.g., $T_{p}>25$ transmit symbols. Moreover, for $T_{p}=13$, the diversity order is lower than $1(d<1)$, i.e., worse than the SU-SISO case.

Figure 5 shows the diversity order computed numerically, as described in Section 4.3, for $f_{\Delta}=1,2$ and $4 \mathrm{ppm}$ 


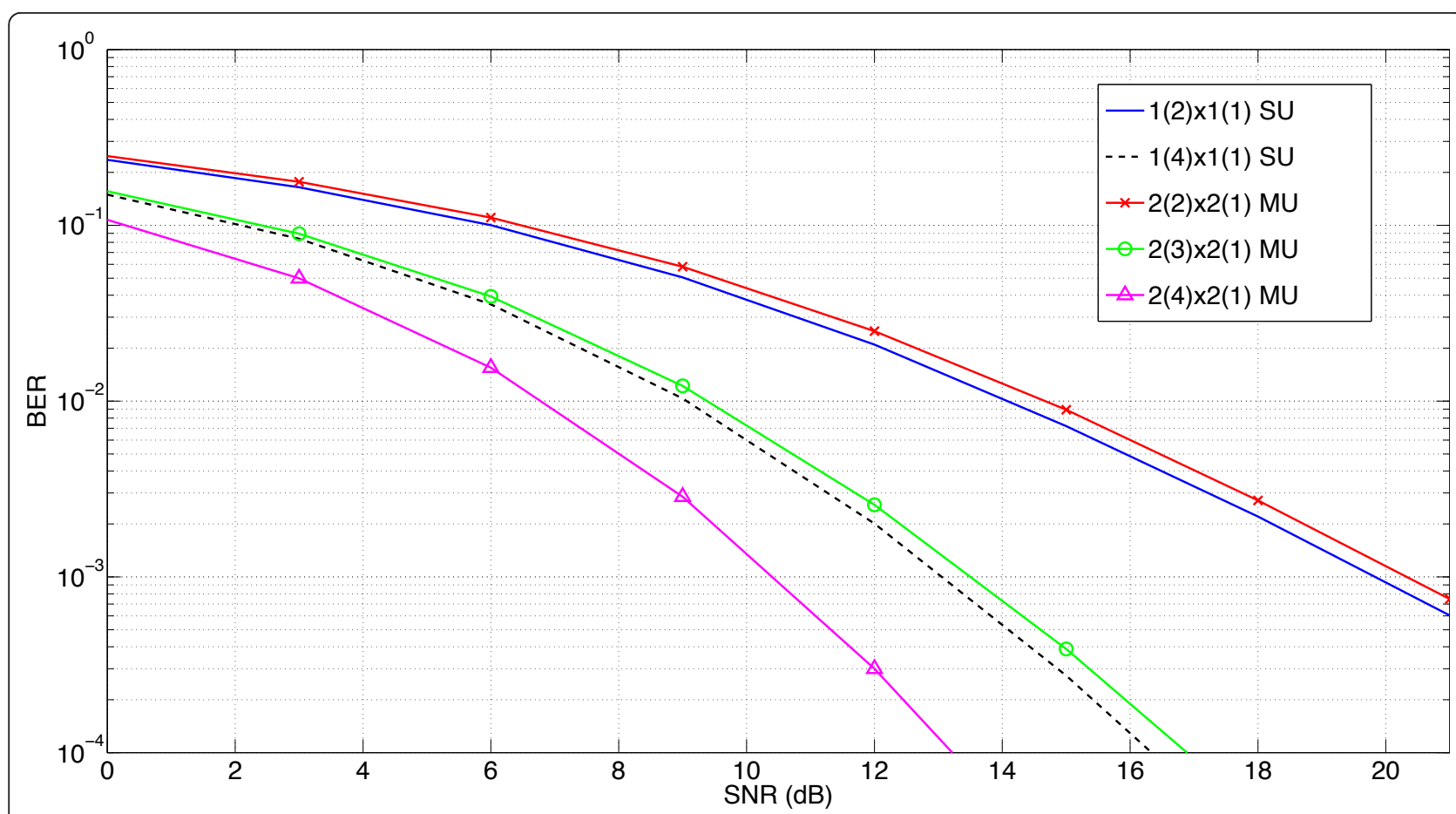

Figure 2 Displays the BER curves versus the SNR, i.e., $1 / \sigma_{n}^{2}$, of the considered ZF beamform-ing scheme, i.e., the multi-user (MU) scenario, with $N_{t}=2$ (continuous red line with the marker $\mathrm{x}$ ), $N_{t}=3$ (continuous green line with the marker o) and $N_{t}=4$ (continuous magenta line with the marker $\Delta$ ) assuming perfect frequency synchronization and for a 16 QAM modulation scheme. We can observe that the diversity order of the proposed distributed schemes increases with the number of transmit antennas, matching the derivations proposed in Section 3.2. The BER curves of a single-user (SU) system with transmit-MRC beamforming and $N_{t}=2$ (continuous blue line) and $N_{t}=$ 4 (dashed black line) are also displayed.

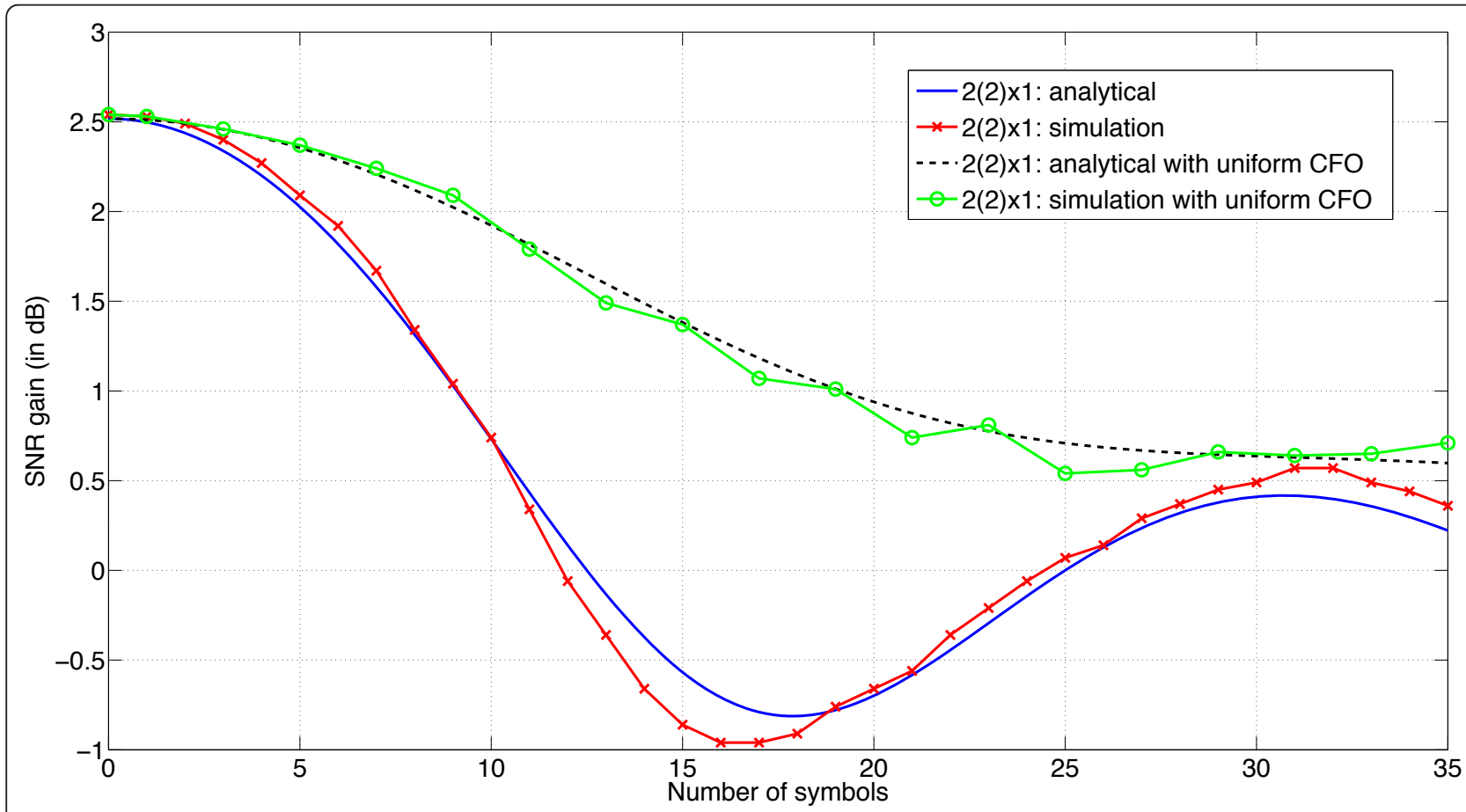

Figure 3 Plot of the analytical and simulated SNR gain for a varying number of transmit symbols, with $f_{\Delta}=2 \mathrm{ppm}$ and the $2(2) \times 2(1)$ scheme. We observe that the SNR gain degradation due to the residual CFO follows a sinc function. The curves for an uniformly distributed CFO are also displayed. The analytical and simulated curves follow the results obtain in Section 4.2, i.e., they have a similar behavior. 


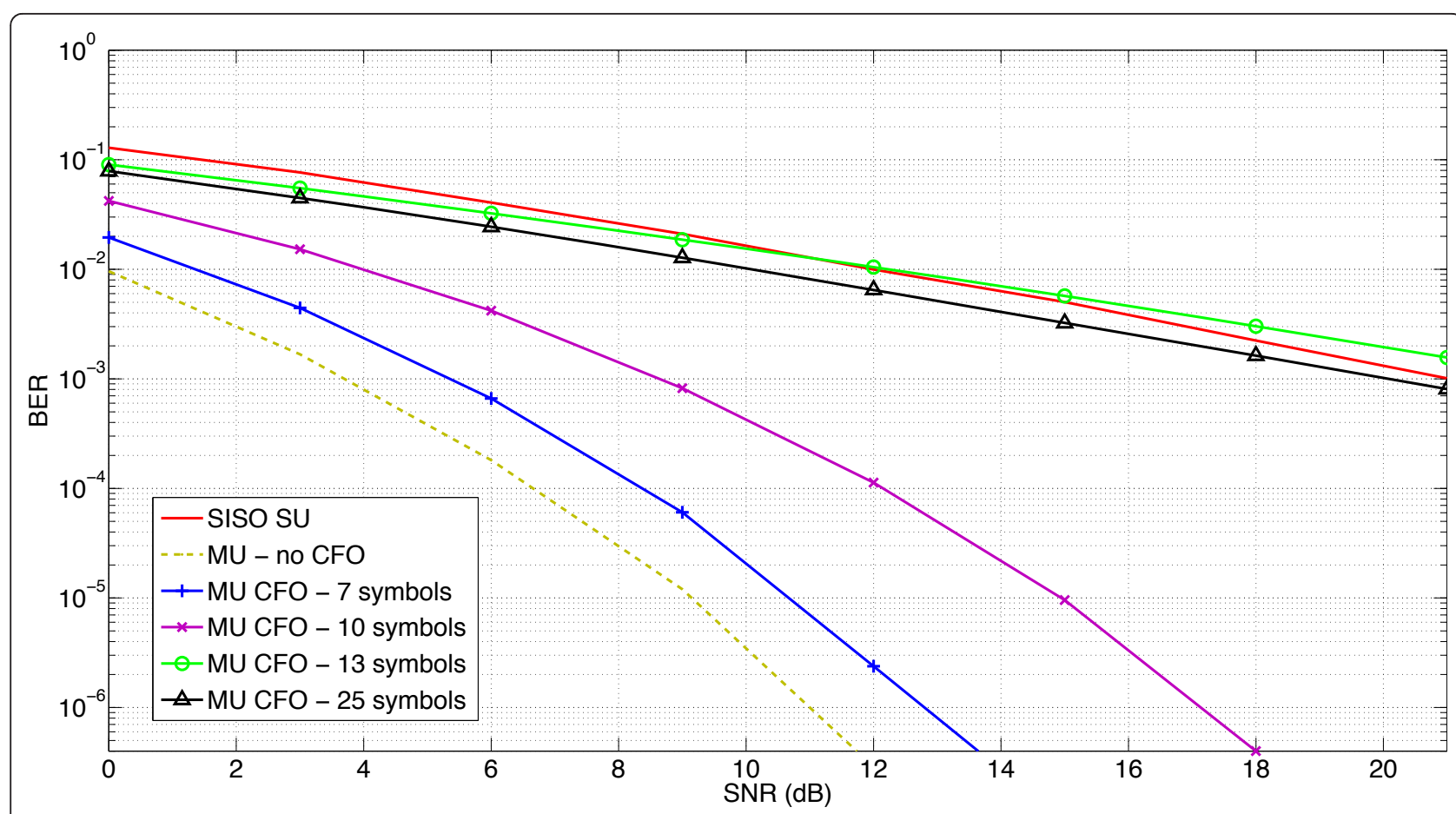

Figure 4 Diversity loss for the $2(3) \times(2) 1$ distributed scenario for $f_{\Delta}=2 \mathrm{ppm}$ for various transmission durations and with a BPSK modulation scheme. From this figure, the diversity order decreases quickly with the number of transmit symbols and the steepness of the curve for $T_{p}=13$ is lower than the SISO curve, i.e., diversity $<1$; confirming the results from Section 4.3.

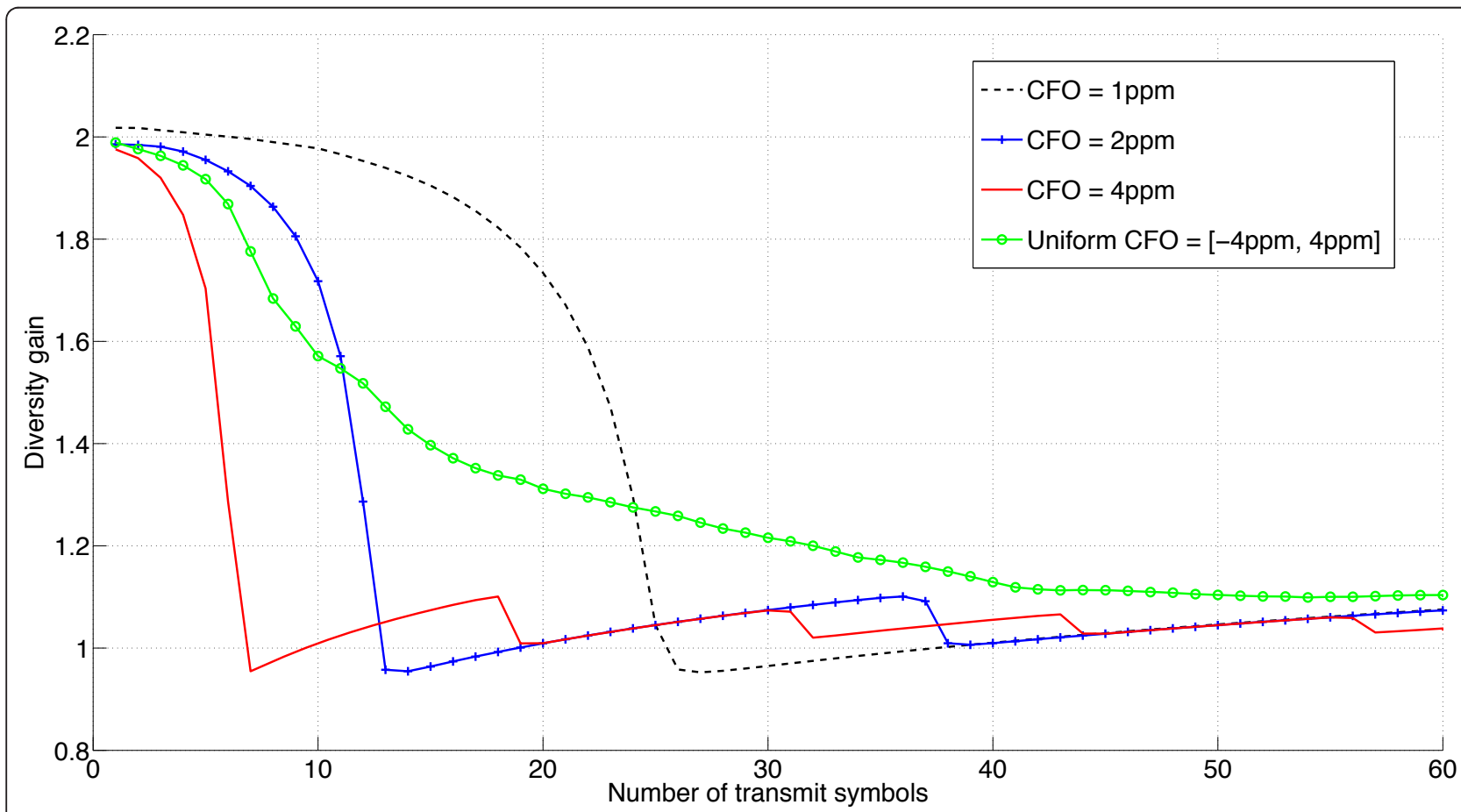

Figure 5 Plot of the numerical diversity order, for various number of transmit symbols and residual CFOs for the $2(2) \times 2(1)$ distributed scenario. From this figure, we can observe that the diversity gain decreases quickly with the residual CFO and the transmission duration. The diversity gain oscillates with the transmission duration and oscillates to converge to the diversity order of a SU-SISO scheme. 
and for a uniformly distributed CFO in the range $f_{\Delta}=$ [-4 ppm, +4 ppm]. We observe that the diversity gain rapidly decreases with the residual CFO to converge around $d=1$, i.e., the diversity gain is lost. Moreover, the figure shows that the degradation of the diversity gain is slower for an uniformly distributed CFO than that for its non-uniform counterpart.

\subsubsection{Tightness of the analytical results}

Figure 6 shows the accuracy of the results from the analytical derivations given in Section 4.3. This figure displays the simulated BER curves (continuous curves) as well as the theoretical ones (dashed line) for $f_{\Delta}=2$ ppm, $N_{t}=2$, a BPSK modulation scheme and for various transmission durations $\left(T_{p}\right)$. From the figure, we can observe that the proposed analytical derivations match the simulated results.

\subsection{Scenario study for $\mathbf{8 0 2 . 1 1}$ and 3GPP-LTE systems}

The Table 1 describes the impacts of CFO, for the considered coordinated scheme, in an IEEE $802.11 \mathrm{n}$ and 3GPP-LTE scenarios with three antennas $\left(N_{t}=3\right)$ at each transmit cell and $P_{1}=P_{2}=1$. The IEEE $802.11 \mathrm{n}$ scenario is as introduced above with 60 symbols burst and no Doppler effect. For such a system, the symbol duration is $3.2 \mu \mathrm{s}$ plus a cyclic prefix of $0.8 \mu \mathrm{s}$ and the accuracy of the CFO estimation is typically of $0.3-1 \mathrm{ppm}$ of the carrier frequency. The 3GPP-LTE system works with a carrier frequency of $2.6 \mathrm{GHz}$ and transmits a burst of seven symbols, with a synchronization error of $25 \mathrm{~Hz}$. One symbol duration is $66.67 \mu \mathrm{s}$ plus a normal cyclic prefix of $5.21 \mu$ s for the first symbol and $4.69 \mu \mathrm{s}$ for the remaining ones, and the CFO is within $0.1 \mathrm{ppm}$ of the carrier frequency [33,34].

Both systems experience a delay between the synchronization stage and the transmission stage, e.g., the synchronization is based on a feedback from the receiver terminal [15]. Therefore, we consider a delay of $50 \mu \mathrm{s}$ for the IEEE802.11 n, e.g., equivalent to the DCF interframe space (DIFS). And a delay of $400 \mu \mathrm{s}$ for the 3GPP-LTE scenario, e.g., because the synchronization occurs within the third OFDM symbol and due to timing advance requirements and control signaling between the transmit cells.

The Table 1 indicates, for representative values of transmit duration, time offset and range of frequency offsets, the SNR and diversity losses in both systems evaluated with Monte Carlo simulations. It shows that the SNR and diversity gains of coordinated beamforming schemes degrade significantly even when the CFO is precompensated before transmission and is worse than a single-user scheme.

\section{Conclusions}

This paper proposes the study of the effects of residual CFO on the ZF distributed beamforming scheme, where

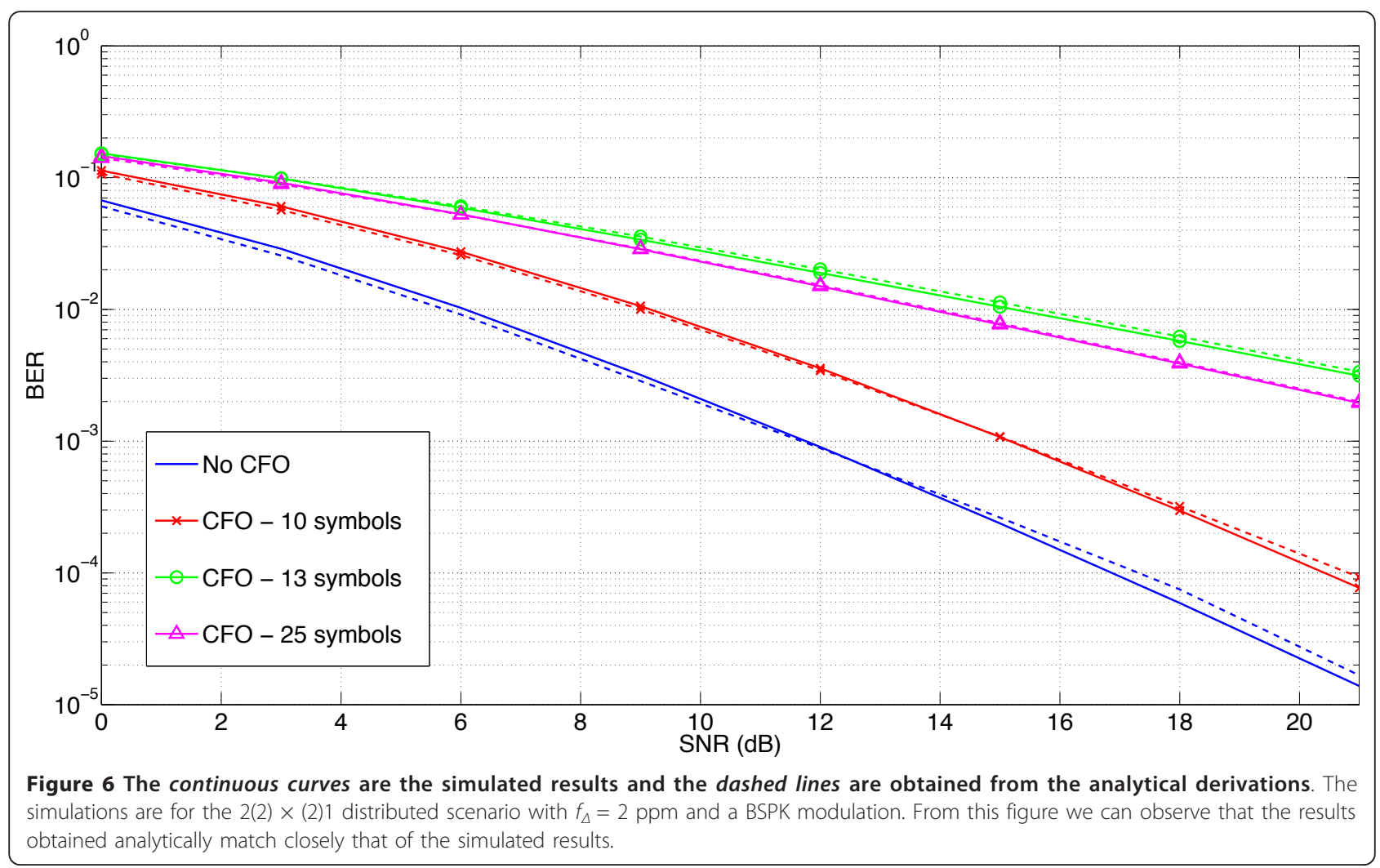


Table 1 Scenario study of the performance degradation due to CFO in coordinated beamforming for the IEEE802.11n and 3GPP-LTE systems

\begin{tabular}{lllll}
\hline System & Timing & CFO $\left(f_{\Delta}\right)$ & SNR loss $(\mathbf{d B})$ & Diversity loss (\%) \\
\hline $802.11 \mathrm{n}$ & $240 \mu \mathrm{s}+50 \mu \mathrm{s}$ & $2-10 \mathrm{kHz}$ & $2.5-3$ & $30-90$ \\
LTE & $500 \mu \mathrm{s}+400 \mu \mathrm{s}$ & $300-600 \mathrm{~Hz}$ & $0.9-2.8$ & $10-60$ \\
\hline
\end{tabular}

both transmitters simultaneously transmit toward two receivers. Analytical derivations and numerical approximations of the SNR and the diversity loss introduced by a fixed and uniform residual CFO, as well as for perfect synchronization, were developed. Results show that, even with a prior-to-transmission synchronization, the residual CFO degrades significantly the SNR and diversity gains. As a result, additional efforts for the estimation and correction of the frequency offset and for reducing the latency between the synchronization stage and the transmission stage are necessary to achieve the gain promised by coordinated transmissions.

\section{Competing interests}

The authors declare that they have no competing interests.

\section{Author details}

${ }^{1}$ Department of Electrical Engineering, Katholieke Universiteit Leuven, Kasteel-park Arenberg 10, B-3001 Leuven, Belgium ${ }^{2}$ Interuniversity MicroElectronics Center (IMEC), Kapeldreef 75, B-3001 Leuven, Belgium ${ }^{3}$ Nanjing University, HanKou Road 22, 210093 Nanjing, P.R. China ${ }^{4}$ Université Libre de Bruxelles, Avenue F. D. Roosevelt 50, B-1050 Brussels, Belgium

Received: 18 March 2011 Accepted: 23 November 2011 Published: 23 November 2011

\section{References}

1. M Sawahashi, Y Kishiyama, A Morimoto, D Nishikawa, M Tanno, Coordinated multipoint transmission/reception techniques for LTEadvanced [Coordinated and Distributed MIMO]. IEEE Wireless Commun. 17(3), 26-34 (2010)

2. D Gesbert, S Hanly, H Huang, S Shamai (Shitz), O Simeone, W Yu, Multi-cell MIMO cooperative networks: a new look at interference. IEEE J Selected Areas Commun. 99, 1-29 (2010)

3. S Sesia, I Toufik, M Baker, LTE-The UMTS Long Term Evolution (Wiley, New York, 2009)

4. BL Ng, JS Evans, SV Hanly, D Aktas, Distributed downlink beamforming with cooperative base stations. IEEE Trans Inf Theory 54(12), 5491-5499 (2008)

5. R Zhang, S Cui, Cooperative interference management with MISO beamforming. IEEE Trans Signal Process. 58(10), 5450-5458 (2010)

6. R Zakhour, D Gesbert, Distributed multicell-MISO precoding using the layered virtual SINR framework. IEEE Trans Wireless Commun. 9(8), 2444-2448 (2010)

7. O Simeone, O Somekh, HV Poor, S Shamai, Distributed MIMO in multi-cell wireless systems via finite-capacity links, in 3rd International Symposium on Communications, Control and Signal Processing (ISCCSP 2008). 203-206 (March, 2008)

8. S Ma, Y Yang, H Sharif, Distributed MIMO technologies in cooperative wireless networks. IEEE Commun Mag. 49(5), 78-82 (2011)

9. Z D Ding, KK Leung, DL Goeckel, D Towsley, On the application of cooperative transmission to wireless broadcast channels, IEEE International Conference on Communications (ICC) (May 2010)

10. F Horlin, A Bourdoux, Digital Compensation for Analog Front-Ends: A New Approach to Wireless Transceiver Design (Wiley, New York, 2008)
11. OS Shin, AM Chan, HT Kung, V Tarokh, Design of an OFDM cooperative space-time diversity system. IEEE Trans Veh Technol. 56(4), 2203-2215 (2007)

12. R Mudumbai, G Barriac, $U$ Madhow, On the feasibility of distributed beamforming in wireless networks. IEEE Trans Wireless Commun. 6(5), 1754-1763 (2007)

13. N Benvenuto, S Tomasin, D Veronesi, Multiple frequency offsets estimation and compensation for cooperative networks, IEEE Wireless Communications and Networking Conference, 891-895 (2007)

14. $\mathrm{X} \mathrm{Li}, \mathrm{F} \mathrm{Ng}, \mathrm{T}$ Han, Carrier frequency offset mitigation in asynchronous cooperative OFDM transmissions. IEEE Trans Signal Process. 56(2), 675-685 (2008)

15. BW Zarikoff, JK Cavers, Coordinated multi-cell systems: carrier frequency offset estimation and correction. IEEE J Selected Areas Commun. 28(9), 1754-1763 (2010)

16. TM Schmidl, DC Cox, Robust frequency and timing synchronization for OFDM. IEEE Trans Commun. 45(12), 1613-1621 (1997). doi:10.1109/ 26.650240

17. Y Zhang, J Zhang, Multiple CFOs compensation and BER analysis for cooperative communication systems, IEEE Wireless Communications and Networking Conference (2009)

18. F Ng, X Li, Cooperative STBC-OFDM transmissions with imperfect synchronization in time and frequency, Asilomar Conference on Signals, Systems and Computers (2005)

19. T Koivisto, V Koivunen, Impact of time and frequency offsets on cooperative multi-user MIMO-OFDM systems. IEEE International Symposium on Personal, Indoor and Mobile Radio Communications, 3119-3123 (2009)

20. B Zarikoff, JK Cavers, Carrier frequency offset in coordinated multibase MIMO narrowband systems, IEEE Vehicular Technology Conference (VTC), 872-877 (2008)

21. H Ochiai, P Mitran, HV Poor, V Tarokh, Collaborative beamforming for distributed wireless ad hoc sensor networks. IEEE Trans Signal Process. 53(11), 4110-4124 (2005)

22. KS Marvin, MS Alouini, Digital Communication over Fading Channels (WileyInterscience, New York, 2000)

23. M Elkashlan, PL Yeoh, RHY Louie, I Collings, On the exact and asymptotic ser of receive diversity with multiple amplify-and-forward relays. IEEE Trans Veh Technol. 59(9), 4602-4608 (2010)

24. M Di Renzo, F Graziosi, F Santucci, A comprehensive framework for performance analysis of dual-hop cooperative wireless systems with fixedgain relays over generalized fading channels. IEEE Trans Wireless Commun. 8(10), 5060-5074 (2009)

25. DB da Costa, S Aissa, Performance of cooperative diversity networks: analysis of amplify-and-forward relaying under equal-gain and maximalratio combining, IEEE International Conference on Communications (2009)

26. K Zarifi, S Zaidi, S Affes, A Ghrayeb, A distributed amplify-and-forward beamforming technique in wireless sensor networks. IEEE Trans Signal Process. 59(8), 3657-3674 (2011)

27. A Paulraj, R Nabar, D Gore, Introduction to Space-Time Wireless Communications (Cambridge University Press, Cambridge, UK, 2008)

28. RA Horn, CR Johnson, Matrix Analysis (Cambridge University Press, Cambridge, UK, 1985)

29. MK Simon, Probability Distributions Involving Gaussian Random Variables (Springer, New York, 2006)

30. PR Krishnaiah, H Peter, S Leon, A note on the bivariate chi distribution. SIAM Rev. 5(2), 140-144 (1963). doi:10.1137/1005034

31. QT Zhang, A simple approach to probability of error for equal gain combiners over Rayleigh channels. IEEE Trans Veh Technol. 48(4), 1151-1154 (1999). doi:10.1109/25.775364

32. IEEE, IEEE Standard for Information technology-Telecommunications and information exchange between systems-Local and metropolitan area networks-Specific requirements Part 11: Wireless LAN Medium Access Control (MAC) and Physical Layer (PHY) Specifications Amendment 5: 
Enhancements for Higher Throughput. IEEE Std 802.11n-2009 (Amendment to IEEE Std 802.11-2007 as amended by IEEE Std $802.11 \mathrm{k}-2008$, IEEE Std 802.11r-2008, IEEE Std 802.11y-2008, and IEEE Std $802.11 \mathrm{w}-2009$ (2009)

33. 3GPP, 3GPP TS36.321 V10.0.0 (E-UTRA); Medium Access Control (MAC) protocol specification (2010)

34. 3GPP, ETSI TS 136211 V9.0.0 (E-UTRA); Physical channels and modulation (2010)

doi:10.1186/1687-1499-2011-177

Cite this article as: Lebrun et al:: Performance analysis of distributed ZF beamforming in the presence of CFO. EURASIP Journal on Wireless Communications and Networking 2011 2011:177.

\section{Submit your manuscript to a SpringerOpen ${ }^{\circ}$ journal and benefit from:}

- Convenient online submission

- Rigorous peer review

- Immediate publication on acceptance

- Open access: articles freely available online

- High visibility within the field

- Retaining the copyright to your article

Submit your next manuscript at $\gg$ springeropen.com 